\section{Efeito da Estratégia Saúde da Família nas internações por condições sensíveis à atenção primária em menores de um ano na Bahia, Brasil}

\author{
Effect of the Family Health Strategy on \\ hospitalizations for primary care sensitive \\ conditions in infants in Bahia State, Brazil
}

Elzo Pereira Pinto Junior 1

Rosana Aquino 1

Maria Guadalupe Medina 1

Marcelo Gurgel Carlos da Silva 2

doi: 10.1590/0102-311X00133816

\title{
Resumo
}

O estudo teve como objetivo avaliar o efeito da expansão da cobertura da Estratégia Saúde da Família (ESF) sobre as internações por condições sensiveis à atenção primária (ICSAP) em crianças menores de um ano. Trata-se de um estudo ecológico longitudinal com uso de painel de dados, cujas unidades de análise foram os 417 municípios da Bahia, Brasil, entre 2000 a 2012. Os dados foram obtidos dos sistemas de informação em saúde. A taxa de ICSAP foi o desfecho e a cobertura da ESF à exposição principal. As covariáveis eram referentes às características demográficas e socioeconômicas e disponibilidade de leitos pediátricos nos municípios. Foi realizada análise de regressão bivariada e multivariada para painel de dados, com resposta binomial negativa e modelos de efeitos fixos, utilizando como medida de associação o risco relativo $(R R)$, bruto e ajustado, com seus respectivos intervalos de confiança. Para controle de efeito de tendência, os modelos foram ajustados pelo tempo. Entre 2000 e 2012, 248.944 ICSAP foram registradas em crianças menores de um ano, e a mediana das taxas municipais de ICSAP reduziu 52,5\% no período, variando de 96,9 para 46,0 hospitalizações evitáveis por 1.000 nascidos vivos. Após o ajuste do modelo, a redução das internações evitáveis se manteve nos diferentes niveis de cobertura da ESF. Este estudo demonstrou os efeitos da consolidação da ESF nas ICSAP em menores de um ano, o que indica a importância do fortalecimento das ações no âmbito da atenção primária, com o intuito de oferecer assistência resolutiva ao primeiro contato e evitar hospitalizações desnecessárias.

Hospitalização; Saúde da Criança; Avaliação do Impacto na Saúde; Atenção Primária à Saúde; Estratégia Saúde da Família

\author{
Correspondência \\ E. P. Pinto Junior \\ Rua Oito de Dezembro 808, apto. 103, Salvador, BA 40150-000, \\ Brasil. \\ elzojr@hotmail.com Salvador, Brasil
2 Programa de Pós-graduação em Saúde Coletiva, Universidade \\ 1 Instituto de Saúde Coletiva, Universidade Federal da Bahia, \\ Estadual do Ceará, Fortaleza, Brasil.
}




\section{Introdução}

A Estratégia Saúde da Família (ESF), eixo ordenador de práticas da atenção primária à saúde no Brasil, busca garantir assistência resolutiva aos problemas de saúde dos usuários do Sistema Único de Saúde (SUS), evitando as hospitalizações desnecessárias. Nesse contexto, os indicadores da atividade hospitalar representam uma medida de efetividade da atenção primária à saúde, permitindo a análise de aspectos da saúde da família pelas condições sensíveis à atenção primária (CSAP). Tais condições representam doenças e agravos para os quais as ações qualificadas e resolutivas da atenção primária à saúde diminuiriam o risco de hospitalizações, de forma que tais hospitalizações ocorram apenas nos casos mais graves $1,2,3,4$.

Os primeiros estudos sobre internações por causas sensíveis à atenção primária (ICSAP), desenvolvidos nos Estados Unidos, nos anos 1990, apontaram que a eliminação de barreiras de acesso aos serviços primários de saúde era responsável pela redução de hospitalizações evitáveis e que a prevalência de maiores taxas de internações por essas causas se concentrava em áreas de pobreza, evidenciando assim as relações entre as barreiras de acesso e os determinantes sociais 1,5. Desde então, diversos países vêm desenvolvendo estudos nessa temática 6, destacando-se Espanha 2, Inglaterra 7 e Canadá 8.

No Brasil, a utilização de indicadores de hospitalizações por causas evitáveis como instrumento de avaliação e monitoramento de efetividade da atenção primária à saúde tem sido crescente após o Ministério da Saúde ter lançado, no ano de 2008, a Lista Brasileira de Condições Sensíveis à Atenção Primária. A lista compreende 19 grupos de causas e é resultado de um trabalho de validação por consenso entre diversos especialistas da área de Saúde Coletiva 4,6.

Ao longo dos anos, a consolidação da ESF tem sido associada à melhoria da qualidade de vida da população, e vários estudos têm demonstrado avanços na redução dos coeficientes de mortalidade infantil e de mortalidade materna, diminuição das taxas de doenças imunopreveníveis e de internações por condições evitáveis 9,10,11,12,13. Ressalta-se ainda que a associação do aumento da cobertura da ESF com a redução de ICSAP acontece em um contexto social, sendo permeada por determinantes sociais da saúde, tais como aspectos socioeconômicos, culturais, ambientais e comportamentais, que influenciam a ocorrência de problemas de saúde, incluindo as internações por causas evitáveis 14,15.

Apesar de crescentes, os estudos brasileiros na temática das hospitalizações evitáveis ainda deixam lacunas no conhecimento, especialmente no tocante às relações entre as hospitalizações e a evolução da cobertura da ESF em estados da Região Nordeste. Dessa forma, este estudo objetiva avaliar o efeito da expansão da cobertura da ESF sobre as ICSAP em crianças menores de um ano, na Bahia, entre os anos 2000 e 2012 .

\section{Métodos}

Trata-se de um estudo ecológico longitudinal com uso de painel de dados (panel data). A escolha por dados em painel permite observar diversas unidades de análise em mais de um ponto no tempo. Neste estudo, as unidades de análise foram os 417 municípios baianos, no período entre 2000 e 2012. A Bahia é um estado localizado na Região Nordeste do Brasil, com população de mais de 15 milhões de habitantes, renda domiciliar per capita da população residente no valor R\$ 697,00 e Índice de Desenvolvimento Humano de 0,660 (Instituto Brasileiro de Geografia e Estatística. http://www. ibge.gov.br/estadosat/perfil.php?sigla=ba, acessado em 20/Abr/2013). No ano de 2013, a cobertura estimada da ESF era de 79,5\% (Secretaria de Saúde do Estado da Bahia. http://www1.saude.ba.gov.br/ mapa_bahia/, acessado em 20/Abr/2013).

Para definição das causas de hospitalizações, foi utilizado o conjunto de 19 causas elencados na lista brasileira de condições sensíveis à atenção primária 4 . As taxas de hospitalização por condições sensíveis à atenção primária à saúde em menores de um ano, variável dependente, foram calculadas dividindo a quantidade dessas hospitalizações por 1.000 crianças nascidas vivas, em cada ano e em cada município analisado. Também foram calculadas as taxas de internação por condições não evitáveis em crianças menores de um ano. Os dados sobre hospitalizações em menores de um ano foram obtidos no Sistema de Informação Hospitalar do SUS (SIH/SUS), e o total de crianças nascidas vivas, do Sistema de Informações sobre Nascidos Vivos (SINASC). 
A variável independente principal foi a cobertura da ESF nos municípios, medida para cada ano, calculada pela razão entre a população coberta pela ESF e a população total do município, limitada a 100\%. As informações de população coberta foram provenientes do Sistema de Informação da Atenção Básica (SIAB), e os dados demográficos, do Instituto Brasileiro de Geografia e Estatística (IBGE). As informações de cobertura foram categorizadas nos seguintes estratos, conforme modelo proposto por Aquino et al. 9: (1) ausente: cobertura igual a 0\%, ou seja, sem implantação da ESF; (2) incipiente: cobertura populacional menor que $30 \%$ e diferente de zero; (3) intermediário: cobertura populacional maior ou igual a $30 \%$ e menor que $70 \%$ ou maior ou igual a $70 \%$ com menos de quatro anos de implantação da ESF; e, (4) consolidado: cobertura populacional maior ou igual a 70\% e com pelo menos, quatro anos de implantação da ESF.

As covariáveis utilizadas foram: disponibilidade de leitos pediátricos; taxa de fecundidade, taxa de analfabetismo, grau de urbanização, Índice de Desenvolvimento Humano Municipal (IDH-M), Índice de Gini, percentual de domicílios com água encanada nesses municípios e renda média domiciliar per capita. As informações acerca da disponibilidade de leitos pediátricos foram obtidas por meio de consulta ao Cadastro Nacional dos Estabelecimentos de Saúde (CNES). Os dados demográficos e socioeconômicos dos municípios foram obtidos do IBGE, sendo referentes apenas aos anos censitários (2000 e 2010). Os valores anuais para os anos intercensitários (2001 a 2009) e para 2011 e 2012 foram estimados por interpolação e extrapolação linear, respectivamente.

$\mathrm{Na}$ análise descritiva das taxas de internação e outras características socioeconômicas e demográficas, utilizou-se a mediana dos indicadores municipais como medida de tendência central, tendo em vista a não normalidade dos dados constatada após o teste de Shapiro-Francia.

Foi realizada análise de regressão bivariada e multivariada para painel de dados, com resposta binomial negativa e modelos de efeitos fixos. Os modelos finais de regressão foram ajustados pela variável "ano calendário", incorporando o componente temporal na análise multivariada. A regressão binomial negativa (RBN), uma adaptação ao modelo de regressão de Poisson, é recomendada para analisar dados numéricos resultante de contagem em situações em que há superdispersão dos dados. A escolha pelo modelo de efeitos fixos foi definida após a aplicação do teste de Hausman. A medida de associação utilizada para estimar o efeito da cobertura da ESF sobre as taxas de ICSAP e hospitalizações não evitáveis em crianças menores de um ano foi o risco relativo, bruto e ajustado pelas covariáveis, com respectivos intervalos de $95 \%$ de confiança (IC95\%). Optou-se por utilizar as hospitalizações não evitáveis para testar a robustez do modelo de regressão.

O software utilizado para a tabulação e processamento do banco de dados foi o TabWin versão 3.6b (http://www.datasus.gov.br), e para a montagem do banco de dados e análise estatística foi utilizado o software Stata, versão 12.0 (StataCorp LP, College Station, Estados Unidos).

A pesquisa foi submetida e aprovada pelo Comitê de Ética em Pesquisa da Universidade Estadual do Ceará (resolução no 678.805, de 30 de abril de 2014).

\section{Resultados}

No período de 2000 a 2012, 248.944 internações por condições sensíveis à Atenção Primária foram registradas em crianças menores de um ano na Bahia. A mediana das taxas municipais de ICSAP reduziu $52,5 \%$ no período, variando de 96,9 para 46,0 hospitalizações evitáveis por 1.000 nascidos vivos. Em relação às medianas das taxas de internações não evitáveis, houve redução de $48,4 \%$ no período, variando de 143,1 para 73,8 hospitalizações não evitáveis por 1.000 nascidos vivos (Figura 1). Nesse mesmo período, a cobertura populacional da ESF na Bahia aumentou de 59,9\% para 79,5\% (Figura 2).

No tocante aos indicadores demográficos e socioeconômicos, foi possível notar melhoria em todas as variáveis investigadas. Destaca-se a redução das taxas de fecundidade e analfabetismo, o aumento no grau de urbanização dos municípios e no percentual de domicílios com água encanada nesses municípios, além do incremento da renda média domiciliar per capita e do IDH-M. Ainda nesse cenário de melhoria de indicadores demográficos e socioeconômicos, aponta-se também a redução do Índice de Gini, que indica melhoria na distribuição de renda. Nos aspectos ligados à oferta de serviços de saúde, notou-se a ampliação do número de municípios com disponibilidade de leitos pediátricos (Tabela 1). 
Figura 1

Mediana das taxas de internações por condições sensíveis à atenção primária (ICSAP) e das taxas de não ICSAP em crianças menores de um ano na Bahia, Brasil, 2000-2012.

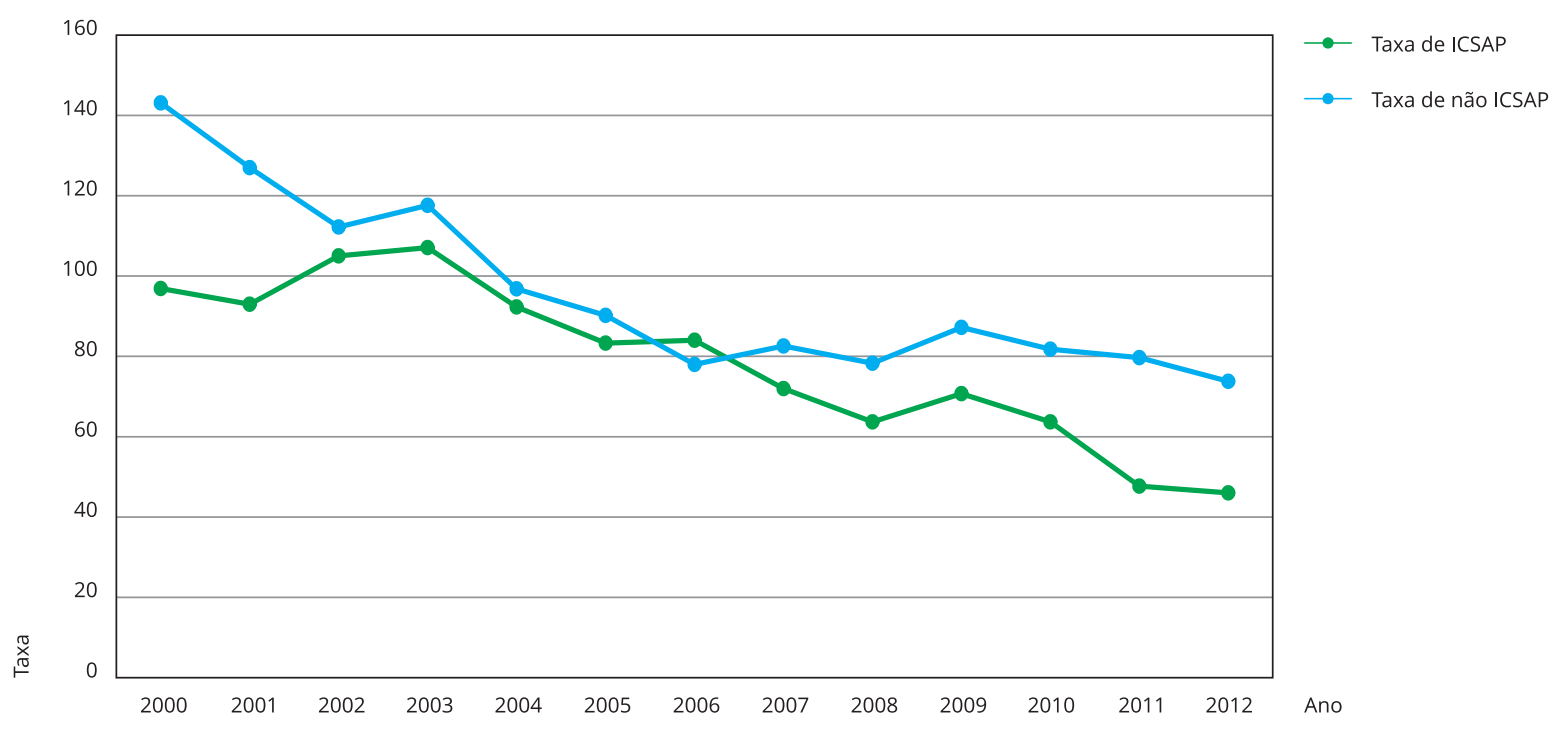

Figura 2

Evolução do percentual de cobertura da Estratégia Saúde da Família (ESF). Bahia, Brasil, 2000-2012.

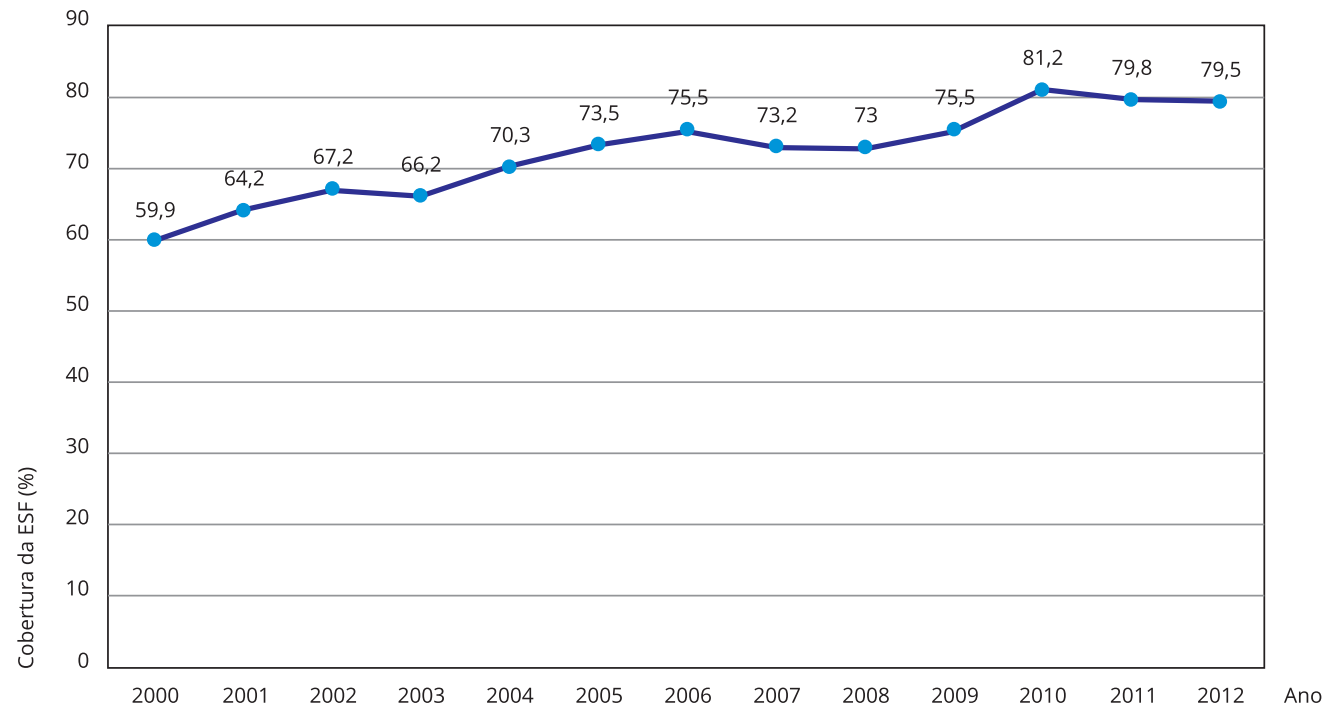


Tabela 1

Características de serviços de saúde e aspectos demográficos e socioeconômicos dos municípios baianos, Brasil, $2000-2012$.

\begin{tabular}{|c|c|c|c|c|c|c|c|c|c|c|c|c|c|}
\hline Variáveis & 2000 & 2001 & 2002 & 2003 & 2004 & 2005 & 2006 & 2007 & 2008 & 2009 & 2010 & 2011 & 2012 \\
\hline Municípios com leitos pediátricos (\%) & 38,8 & 41,0 & 42,4 & 46,8 & 48,9 & 48,7 & 50,1 & 49,9 & 49,1 & 53,5 & 53,7 & 56,6 & 56,6 \\
\hline Taxa de fecundidade * & 3,0 & 2,9 & 2,8 & 2,7 & 2,7 & 2,6 & 2,5 & 2,5 & 2,4 & 2,4 & 2,3 & 2,2 & 2,1 \\
\hline Taxa de analfabetismo * & 30,6 & 29,8 & 29,3 & 28,6 & 28,1 & 27,5 & 26,9 & 26,2 & 25,5 & 25,0 & 24,1 & 23,4 & 22,7 \\
\hline Grau de urbanização * & 46,0 & 46,9 & 47,6 & 48,0 & 48,8 & 49,3 & 49,5 & 50,0 & 50,4 & 51,3 & 51,4 & 52,0 & 52,1 \\
\hline Domicílios com água encanada (\%) * & 43,2 & 46,4 & 50,0 & 53,6 & 56,9 & 60,8 & 64,7 & 68,0 & 71,6 & 75,6 & 79,2 & 83,0 & 86,8 \\
\hline Renda média domiciliar per capita $(\mathrm{R} \$)$ * & 165,9 & 176,0 & 187,1 & 198,6 & 209,4 & 219,3 & 229,1 & 239,1 & 248,7 & 256,8 & 266,4 & 275,6 & 285,3 \\
\hline Índice de Gini * & 0,568 & 0,567 & 0,565 & 0,561 & 0,556 & 0,552 & 0,549 & 0,545 & 0,543 & 0,539 & 0,537 & 0,534 & 0,529 \\
\hline IDH-M * & 0,422 & 0,439 & 0,456 & 0,473 & 0,488 & 0,505 & 0,521 & 0,539 & 0,555 & 0,572 & 0,589 & 0,605 & 0,622 \\
\hline
\end{tabular}

IDH-M: Índice de Desenvolvimento Humano-Municipal.

Fonte: dados da pesquisa (2014).

* Medianas com base nos dados de cada um dos municípios.

O modelo bruto da análise de regressão demonstrou que a implantação da ESF em municípios baianos reduziu as taxas de ICSAP em menores de um ano, mas naqueles locais cuja ESF foi considerada consolidada essa redução chegou a 38\%. Quando o modelo foi ajustado pelas covariáveis, incluindo a variável para controle do efeito temporal, a redução das internações evitáveis se manteve presente nos diferentes níveis de cobertura, seja ela incipiente $(R R=0,68$; IC95\%: 0,56-0,83), intermediária $(R R=0,80$; IC95\%: 0,66-0,96) ou consolidada ( RR = 0,81; IC95\%: 0,67-0,97). Já nas internações por causas não evitáveis, o efeito de redução nas ICSAP foi observado apenas nos municípios cuja cobertura era incipiente $(\mathrm{RR}=0,72$; IC95\%: 0,61-0,86) (Tabela 2).

\section{Discussão}

Os resultados deste estudo mostram que a expansão na cobertura da ESF nos municípios da Bahia, entre 2000 e 2012, esteve associada à significativa redução nas ICSAP em crianças menores de um ano. A queda das hospitalizações por este grupo de causas aconteceu num cenário marcado pela expansão da atenção primária à saúde na Bahia, evidenciado pelo aumento da cobertura da ESF. Destaca-se a magnitude dessa redução nos três estratos de cobertura, mesmo após o ajuste pelo tempo. Além disso, neste mesmo período, foi verificada ampliação do acesso a leitos hospitalares pediátricos e melhoria nos indicadores demográficos e socioeconômicos dos municípios baianos.

O efeito inesperado de maior magnitude no estrato de cobertura incipiente pode ser explicado pelo fato de que a maioria dos municípios com cobertura inferior a 30\% eram de grande porte populacional. Nesses cenários, mesmo um baixo percentual da ESF, pode produzir efeito nas ICSAP, uma vez que essa cobertura concentra-se nas áreas mais vulneráveis do município, o que pode resultar numa diminuição das desigualdades no acesso a serviços de saúde ao incorporar à rede de atenção à saúde áreas que anteriormente estavam desprovidas desses serviços.

Uma possível hipótese para a associação da cobertura da ESF com a redução das taxas de internações por causas não evitáveis, que foi significante no estrato de cobertura incipiente, relaciona-se com o elenco de diagnóstico da lista brasileira de condições sensíveis à atenção primária à saúde. Sendo uma lista única para todas as faixas etárias, não há aprofundamento para o grupo de menores de um ano e algumas condições que poderiam ser evitáveis pela ação de serviços de atenção primária à saúde, poderiam não estar contempladas. Quando comparada com a lista de causas de mortes evitáveis por intervenções do SUS, constata-se que parte das condições descritas especialmente no grupo de causas "reduzíveis por ações adequadas de promoção à saúde, vinculadas a ações adequadas de atenção à 


\section{Tabela 2}

Modelos de efeitos fixos, bruto e ajustado, para análise entre as taxas de internações por condições sensíveis à atenção primária (ICSAP) e internações não evitáveis e cobertura da Estratégia Saúde da Família (ESF), controlados por características demográficas e socioeconômicas, em crianças menores de um ano, dos municípios da Bahia, Brasil, 2000-2012.

\begin{tabular}{|c|c|c|c|}
\hline \multirow[t]{2}{*}{ Variáveis } & \multicolumn{2}{|c|}{ Taxa de ICSAP (IC95\%) } & \multirow{2}{*}{$\begin{array}{c}\text { Taxa de internações não } \\
\text { evitáveis (IC95\%) }\end{array}$} \\
\hline & RR bruto (IC95\%) & RR ajustado (IC95\%) & \\
\hline \multicolumn{4}{|l|}{ Cobertura ESF } \\
\hline Sem cobertura & 1,00 & 1,00 & 1,00 \\
\hline Incipiente & $0,79(0,65-0,96)$ & $0,68(0,56-0,83)$ & $0,72(0,61-0,86)$ \\
\hline Intermediária & $0,80(0,67-0,96)$ & $0,80(0,66-0,96)$ & $0,91(0,77-1,07)$ \\
\hline Consolidada & $0,62(0,51-0,75)$ & $0,81(0,67-0,97)$ & $0,94(0,80-1,11)$ \\
\hline Taxa de fecundidade $>$ mediana & & $1,00(0,95-1,04)$ & $0,95(0,91-0,99)$ \\
\hline Renda per capita $>$ mediana & & $0,93(0,89-0,98)$ & $0,92(0,88-0,96)$ \\
\hline Taxa de analfabetismo > mediana & & $1,14(1,09-1,20)$ & $1,16(1,11-1,21)$ \\
\hline Percentual de domicílios com água encanada > mediana & & $0,94(0,89-0,98)$ & $0,85(0,82-0,88)$ \\
\hline Índice de Gini > mediana & & $0,89(0,86-0,93)$ & $0,92(0,89-0,96)$ \\
\hline Presença de leitos pediátricos do SUS & & $0,98(0,96-1,03)$ & $0,98(0,93-1,03)$ \\
\hline Ano-calendário & & $0,97(0,96-0,97)$ & $0,97(0,96-0,97)$ \\
\hline
\end{tabular}

IC95\%: intervalo de 95\% de confiança; RR: risco relativo; SUS: Sistema Único de Saúde.

Fonte: dados da pesquisa (2014).

* Teste de Hausman (fixed vs. random): valor de $\mathrm{p}<0,001$.

saúde”, não consta na lista brasileira de condições sensíveis à atenção primária ${ }^{16}$. Destaca-se que as intervenções de SUS no âmbito da promoção da saúde se operacionalizam nos cenários da atenção primária à saúde, o que poderia explicar a associação encontrada neste estudo.

Estudo realizado sobre a tendência das hospitalizações por causas evitáveis no Brasil, utilizando desenho ecológico com dados em painel, ressaltou maior redução das ICSAP na maioria dos estados e regiões, sendo esse resultado atribuído à expansão da ESF 11. Achados semelhantes foram descritos em estudos ecológicos que analisaram tanto o Brasil, segundo estados e regiões 17 , quanto coordenadorias de saúde ou municípios de determinada Unidade Federativa 18,19,20, e também evidenciaram associação entre redução das internações evitáveis e ampliação da cobertura da ESF. Tais resultados corroboram os achados de uma revisão sistemática conduzida por Rosano et al. 21, que confirma a relação entre os indicadores de acesso à atenção primária à saúde e as hospitalizações por condições sensíveis, mostrando menores internações por essas condições em áreas com maior cobertura da atenção primária à saúde.

A redução das ICSAP em grupos etários compostos por menores de cinco anos, que inclui a faixa etária escolhida neste trabalho, também tem sido evidenciada em alguns estudos ecológicos cujas unidades de análise são municípios da Região Nordeste. Pesquisas conduzidas no Piauí e em Pernambuco, que analisaram a relação entre as taxas de ICSAP em crianças e a ESF revelaram que o declínio dessas hospitalizações pode ser atribuído à consolidação da cobertura da atenção primária à saúde 22,23. Não foram encontrados estudos que analisassem especificamente o grupo de menores de um ano, sendo esta uma importante contribuição deste trabalho.

A redução significativa das taxas de hospitalização em menores de um ano pode ser explicada pelo fato de este grupo ser alvo de políticas de saúde mais consolidadas, como o Programa Nacional de Imunização (PNI) e as rotinas de acompanhamento à gestante, à mãe e ao bebê nos primeiros meses de vida, desenvolvidas na atenção primária à saúde. Os fatores demográficos e socioeconômicos, como a melhoria do saneamento básico, o aumento da urbanização e a queda na fecundidade ainda sugerem um cenário socioambiental que favorece a melhoria do cuidado à saúde da criança 24 . 
Além dos estudos que investigam o conjunto de causas de internações evitáveis, pesquisas que avaliam o comportamento de causas específicas de ICSAP, como gastroenterites e pneumonias, também dão suporte às avaliações da efetividade da ESF com base em indicadores de hospitalização. Um estudo sobre o impacto do Programa Saúde da Família (PSF) nas gastroenterites em crianças em 12 municípios na Bahia 25 demonstrou efeito estatisticamente significante nos modelos bruto e ajustado por IDH. Entretanto, no modelo mais completo, que incluiu, além do IDH, serviços de coleta de lixo, de saneamento básico e disponibilidade de água potável, apesar de a medida de associação indicar uma associação negativa entre PSF e hospitalizações por gastroenterites, a associação não foi estatisticamente significante.

Apesar da análise dos efeitos dos determinantes sociais não ter sido o foco deste estudo, foi possível notar que a redução das ICSAP e a expansão da ESF ocorrem num cenário marcado por melhoria nos indicadores demográficos e socioeconômicos municipais, como renda, urbanização, escolaridade e IDH. Estudos específicos sobre o efeito desses determinantes sociais nas ICSAP demonstram que o melhor desempenho desses indicadores se reflete em diminuição nas taxas de internações evitáveis em indivíduos menores de cinco anos 26,27 e em outras populações, considerando adultos e idosos 28,29,30. Neste estudo, a inclusão desse conjunto de variáveis nos modelos de regressão controlou o efeito das características demográficas e socioeconômicas dos municípios e permitiu obter uma medida aproximada do efeito isolado da associação entre os níveis de consolidação da ESF e as ICSAP, garantindo maior robustez à análise.

A análise do efeito da ESF sobre as hospitalizações por condições sensíveis demonstra o impacto desse modelo de organização da atenção primária à saúde na melhoria das condições de saúde em crianças menores de um ano. Acredita-se que o mecanismo explicativo desse efeito seja semelhante ao descrito por Aquino et al. 9, que, ao analisarem o impacto do PSF na mortalidade infantil em municípios brasileiros, com métodos semelhantes ao utilizados neste trabalho, evidenciaram associação entre o nível de implantação do PSF e indicadores de serviços de atenção primária à saúde, demonstrando que a consolidação do PSF se traduziu no aumento de consultas básicas e de atividades educativas por habitante, no maior percentual de grávidas com pelo menos uma consulta de pré-natal e em coberturas maiores de programas de vacinação destinadas ao público infantil. Esse conjunto de evidências reforça o papel da atenção primária à saúde na melhoria das condições de saúde da população materno-infantil coberta pelas equipes de saúde da família.

Os estudos ecológicos têm alcançado um grau de destaque no cenário da avaliação de impacto de políticas públicas, em especial na área da Saúde Coletiva no Brasil. Muitos desses avanços se devem à utilização de métodos estatísticos mais robustos nas pesquisas em saúde. Chama atenção a aplicação dos dados em painel em investigações com dados secundários provenientes dos sistemas de informação em saúde do SUS e de outros órgãos públicos brasileiros, como o IBGE e o Instituto de Pesquisa Econômica Aplicada (IPEA).

A utilização da faixa etária composta por crianças menores de um ano de idade se deu pelo fato de esses indivíduos serem usuários frequentes da atenção primária estarem mais suscetíveis ao adoecimento pelas doenças presentes na lista brasileira de ICSAP e por suas condições de saúde sofrerem influência direta da oferta de serviços de saúde e dos determinantes sociais da saúde.

A escolha por estudos ecológicos com uso de sistemas de informação em saúde considerou a disponibilidade na obtenção dos dados, o baixo custo e a rapidez na execução de um estudo com o objetivo de avaliar diversos aspectos de 417 municípios num período de 13 anos. Mesmo com problemas de subnotificação e erros de preenchimentos dos registros, têm sido encontrados nos últimos anos avanços significativos na qualidade dos sistemas de informação em saúde, fato que permite a realização de estudos ecológicos robustos 31 . Destaca-se ainda como uma limitação que deve ser trazida para o bojo da discussão dessa temática a unicidade da lista brasileira de condições sensíveis à atenção primária à saúde. Este documento, que considera todas as causas evitáveis para todas as faixas etárias, não leva em conta as peculiaridades na ocorrência de doenças em crianças, adultos e idosos. Esse debate também está presente no cenário nacional, haja vista a necessidade de ajustes adicionais na definição das condições evitáveis de hospitalização para o monitoramento da atenção primária 32.

Em que pese a importância do indicador "internações por condições sensíveis à atenção primária" e a sua utilidade como medida indireta de resolubilidade/qualidade da atenção básica, faz-se necessária a adequação dos códigos da lista brasileira de condições sensíveis à atenção primária de acordo 
com os estratos populacionais, de modo semelhante ao que acontece com a lista brasileira de mortes evitáveis por intervenções do SUS 33. Dessa forma, com listas específicas para crianças, adultos e idosos, os efeitos das ações no cuidado primário poderão ser medidos com mais precisão, ordenando, de maneira mais rápida e eficiente, a gestão e o planejamento das ações e serviços de saúde.

Este estudo demonstrou os efeitos da consolidação das ações no nível da atenção primária, na melhoria das condições de saúde das crianças menores de um ano. Ressalta-se a importância do fortalecimento das ações em saúde no âmbito da ESF, com o intuito de ofertar assistência resolutiva ao primeiro contato e evitar hospitalizações desnecessárias.

Apesar dos avanços teóricos e metodológicos evidenciados neste estudo, investigações no âmbito nacional são importantes para compreender a relação entre a expansão da cobertura dos serviços de atenção primária à saúde e as internações evitáveis em menores de um ano em cenários mais abrangentes. Além disso, frisa-se que estudos individuados de base populacional, como estudos transversais ou de coorte, podem ajudar na compreensão de outros determinantes das ICSAP.

\section{Colaboradores}

E. P. Pinto Junior contribuiu com a concepção e projeto, análise e interpretação dos dados, redação do artigo e aprovação final da versão a ser publicada. R. Aquino contribuiu com a análise e interpretação dos dados, revisão crítica relevante do conteúdo intelectual e aprovação final da versão a ser publicada. M. G. Medina contribuiu com a interpretação dos dados, revisão crítica relevante do conteúdo intelectual e aprovação final da versão a ser publicada. M. G. C. Silva contribuiu com a concepção e projeto, interpretação dos dados, revisão crítica relevante do conteúdo intelectual e aprovação final da versão a ser publicada.

\section{Agradecimentos}

À Coordenação de Aperfeiçoamento de Pessoal de Nível Superior (Capes) e ao Conselho Nacional de Desenvolvimento Científico e Tecnológico (CNPq) pela concessão das bolsas de estudo para pós-graduação (mestrado e doutorado). 


\section{Referências}

1. Billings J, Teicholz N. Uninsured patients in District of Columbia hospitals. Health Aff (Millwood) 1990; 9:158-65.

2. Caminal Homar J, Casanova Matutano C. La evaluación de la atención primaria y las hospitalizaciones por ambulatory care sensitive conditions. Marco conceptual. Aten Prim 2003; 31:61-5.

3. Caminal J, Starfield B, Sanchéz E, Casanova C, Morales M. The role of primary care in preventing ambulatory care sensitive conditions. Eur J Public Health 2004; 14:246-51.

4. Alfradique ME, Bonolo PDF, Dourado I, Lima-Costa MF, Macinko J, Mendonça CS, et al. Internações por condições sensíveis à atenção primária: a construção da lista brasileira como ferramenta para medir o desempenho do sistema de saúde (Projeto ICSAP - Brasil). Cad Saúde Pública 2009; 25:1337-49.

5. Billings J, Zeitel L, Lukomnik J, Carey TS, Blank AE, Newman L. Impact of socioeconomic status on hospital use in New York City. Health Aff (Millwood) 1993; 12:162-73.

6. Nedel FB, Facchini LA, Martín M, Navarro A Características da atenção básica associadas ao risco de internar por condições sensíveis à atenção primária: revisão sistemática da literatura. Epidemiol Serv Saúde 2010; 19:61-75.

7. Harrison MJ, Dusheiko M, Sutton M, Gravelle H, Doran T, Roland M. Effect of a national primary care pay for performance scheme on emergency hospital admissions for ambulatory care sensitive conditions: controlled longitudinal study. BMJ 2014; 349:g6423.

8. Roos LL, Walld R, Uhanova J, Bond R. Physician visits, hospitalizations, and socioeconomic status: ambulatory care sensitive conditions in a Canadian setting. Health Serv Res 2005; 40:1167-85.

9. Aquino R, Oliveira NF, Barreto ML. Impact of the family health program on infant mortality in Brazilian municipalities. Am J Public Health 2009; 99:87-93.
10. Rasella D, Aquino R, Barreto ML. Impact of the Family Health Program on the quality of vital information and reduction of child unattended deaths in Brazil: an ecological longitudinal study. BMC Public Health 2010; 10:380.

11. Dourado I, Oliveira VB, Aquino R, Bonolo P, Lima-Costa, MF, Medina MG, et al. Trends in primary health care-sensitive conditions in Brazil: the role of the Family Health Program (Project ICSAP-Brazil). Med Care 2011; 49:577-84.

12. Paim J, Travassos C, Almeida C, Bahia L, Macinko J. The Brazilian health system: history, advances, and challenges. Lancet 2011; 377:1778-97.

13. Victora CG, Aquino EM, Leal MC, Monteiro CA, Barros FC, Szwarcwald CL. Maternal and child health in Brazil: progress and challenges. Lancet 2011; 377:1863-76.

14. Ansari Z, Haider SI, Ansari H, De Gooyer T, Sindall C. Patient characteristics associated with hospitalisations for ambulatory care sensitive conditions in Victoria, Australia. BMC Health Serv Res 2012; 12:475.

15. Falster MO, Jorm LR, Douglas KA, Blyth FM, Elliott RF, Leyland AH. Sociodemographic and health characteristics, rather than primary care supply, are major drivers of geographic variation in preventable hospitalizations in Australia. Med Care 2015; 53:436-45.

16. Malta DC, Sardinha LMV, Moura L, Lansky S, Leal MC, Szwarcwald CL, et al. Atualização da lista de causas de mortes evitáveis por intervenções do Sistema Único de Saúde do Brasil. Epidemiol Serv Saúde 2010; 19:173-6.

17. Boing AF, Vicenzi RB, Magajewski F, Boing AC, Moretti-Pires RO, Peres KG, et al. Redução das internações por condições sensíveis à atenção primária no Brasil entre 1998-2009. Rev Saúde Pública 2012; 46:359-66.

18. Rehem TCMSB, Egry EY. Internações por condições sensíveis à atenção primária no estado de São Paulo. Ciênc Saúde Coletiva 2011; 16:4755-66. 
19. Souza LL, Costa JSD. Internações por condições sensíveis à atenção primária nas coordenadorias de saúde no RS. Rev Saúde Pública 2011; 45:765-72.

20. Ceccon RF, Meneghel SN, Viecili PRN. Internações por condições sensíveis à atenção primária e ampliação da Saúde da Família no Brasil: um estudo ecológico. Rev Bras Epidemiol 2014; 17:968-77.

21. Rosano A, Loha CA, Falvo R, Van Der Zee J, Ricciardi W, Guasticchi, G, et al. The relationship between avoidable hospitalization and accessibility to primary care: a systematic review. Eur J Public Health 2012; 23:356-60.

22. Barreto JOM, Nery IS, Costa MSC. Estratégia Saúde da Família e internações hospitalares em menores de 5 anos no Piauí, Brasil. Cad Saúde Pública 2012; 28:515-26.

23. Carvalho SC, Mota E, Dourado I, Aquino R, Teles C, Medina MG. Hospitalizations of children due to primary health care sensitive conditions in Pernambuco State, Northeast Brazil. Cad Saúde Pública 2015; 31:744-54.

24. Moura BLA, Cunha RCD, Aquino R, Medina MG, Mota ELA, Macinko J, et al. Principais causas de internação por condições sensíveis à atenção primária no Brasil: uma análise por faixa etária e região. Rev Bras Saúde Matern Infant 2010; 10 Suppl 1:s83-91.

25. Monahan LJ, Calip GS, Novo PM, Sherstinsky M, Casiano, M, Mota E, et al. Impact of the Family Health Program on gastroenteritis in children in Bahia, Northeast Brazil: an analysis of primary care-sensitive conditions. J Epidemiol Glob Health 2013; 3:175-85.

26. Agha MM, Glazier RH, Guttmann A. Relationship between social inequalities and ambulatory care-sensitive hospitalizations persists for up to 9 years among children born in a major Canadian Urban Center. Ambul Pediatr 2007; 7:258-62.
27. Ricketts TC, Randolph R, Howard HA, Pathman D, Carey T. Hospitalization rates as indicators of access to primary care. Health Place 2001; 7:27-38.

28. Berlin C, Busato A, Rosemann T, Djalali S, Maessen M. Avoidable hospitalizations in Switzerland: a small area analysis on regional variation, density of physicians, hospital supply and rurality. BMC Health Serv Res 2014; 14:289.

29. Gao J, Moran E, Li YF, Almenoff PL. Predicting potentially avoidable hospitalizations. Med Care 2014; 52:164-71.

30. Laditka JN. Hazards of hospitalization for ambulatory care sensitive conditions among older women: evidence of greater risks for African Americans and Hispanics. Med Care Res Rev 2003; 60:468-95.

31. Bittencourt SA, Camacho LAB, Leal MC. O Sistema de Informação Hospitalar e sua aplicação na saúde coletiva. Cad Saúde Pública 2006; 22:19-30.

32. Eggli Y, Desquins B, Seker E, Halfon P. Comparing potentially avoidable hospitalization rates related to ambulatory care sensitive conditions in Switzerland: the need to refine the definition of health conditions and to adjust for population health status. BMC Health Serv Res 2014; 14:25.

33. Malta DC, Duarte EC, Almeida MF, Dias MAS, Morais Neto OL, Moura L, et al. Lista de causas de mortes evitáveis por intervenções do Sistema Único de Saúde do Brasil. Epidemiol Serv Saúde 2007; 16:233-44. 


\section{Abstract}

This study aimed to assess the effect of the expansion of the Family Health Strategy (FHS) on hospitalizations for primary care sensitive conditions (PCSCs) in children under one year of age. This was a longitudinal ecological study with the use of panel data, for which the analytical units were the 417 municipalities (counties) in Bahia State, Brazil, from 2000 to 2012. Data were obtained from the official health information systems. The hospitalization rate for PCSCs was the outcome and FHS coverage was the principal exposure. The co-variables referred to demographic and socioeconomic characteristics and the local availability of pediatric beds. Bivariate and multivariate panel data analyses were performed, with negative binomial response and fixed effects models, using crude and adjusted relative risk $(R R)$ as the measure of association, with the respective confidence intervals. To control for trend effect, the models were adjusted for time. From 2000 to 2012, 248,944 hospitalizations for PCSCs were recorded in children under one year, and the median municipal rate of hospitalizations for PCSCs decreased by $52.5 \%$ during the period, ranging from 96.9 to 46.0 avoidable hospitalizations per 1,000 live births. After adjusting the model, the reduction in avoidable hospitalizations was maintained at the different FHS coverage levels. This study demonstrated the effects of the consolidation of the FHS on hospitalizations for PCSCs in infants, which indicates the importance of strengthening primary care measures in order to offer case-resolving care during the first contact with the health system and avoid unnecessary hospitalizations.

Hospitalization; Child Health; Health Impact Assessment; Primary Health Care;

Family Health Strategy

\section{Resumen}

El objetivo del estudio fue evaluar el efecto de la expansión de la cobertura de la Estrategia Salud de la Familia (ESF) sobre los internamientos por condiciones sensibles en la atención primaria (ICSAP) con niños menores de un año. Se trata de un estudio ecológico longitudinal, con uso de panel de datos, cuyas unidades de análisis fueron los 417 municipios del Estado de Bahía, Brasil, del 2000 al 2012. Los datos se obtuvieron de los sistemas de información en salud. La tasa de ICSAP fue el desenlace y la cobertura de la ESF a la exposición principal. Las covariables se referían a las características demográficas y socioeconómicas y a la disponibilidad de camas pediátricas en los municipios. Se realizó un análisis de regresión bivariado y multivariado para el panel de datos, con una respuesta binomial negativa y modelos de efectos fijos, utilizando como medida de asociación el riesgo relativo (RR), bruto y ajustado, con sus respectivos intervalos de confianza. Para el control de efecto de tendencia, los modelos se ajustaron por el tiempo. Entre 2000 y 2012, 248.944 ICSAP se registraron en niños menores de un año, y la media de las tasas municipales de ICSAP se redujo un 52,5\% en el periodo, variando de 96,9 a 46,0 hospitalizaciones evitables por 1.000 nacidos vivos. Tras el ajuste del modelo, la reducción de los internamientos evitables se mantuvo en los diferentes niveles de cobertura de la ESF. Este estudio demostró los efectos de la consolidación de la ESF en las ICSAP en menores de un año, lo que indica la importancia del fortalecimiento de las acciones en el ámbito de la atención primaria, con la intención de ofrecer asistencia resolutiva con el primer contacto y evitar hospitalizaciones desnecesarias.

Hospitalización; Salud del Niño; Evaluación del Impacto en la Salud; Atención Primaria de Salud; Estrategia de Salud Familiar
Recebido em 29/Jul/2016

Versão final reapresentada em 20/Jun/2017

Aprovado em 05/Jul/2017 